\title{
La comparaison des langues : modèles et directions
}

Jean Rousseau

\section{OpenEdition}

1 Journals

Édition électronique

URL : http://journals.openedition.org/ries/4309

DOI : 10.4000/ries.4309

ISSN : 2261-4265

Éditeur

Centre international d'études pédagogiques

Édition imprimée

Date de publication : 1 mars 1994

Pagination : 97-106

ISSN : 1254-4590

\section{Référence électronique}

Jean Rousseau, "La comparaison des langues : modèles et directions ", Revue internationale

d'éducation de Sèvres [En ligne], 01 | 1994, mis en ligne le 20 avril 2015, consulté le 01 mai 2019. URL http://journals.openedition.org/ries/4309; DOI : 10.4000/ries.4309

Ce document a été généré automatiquement le 1 mai 2019.

(c) Tous droits réservés 


\title{
La comparaison des langues : modèles et directions
}

\author{
Jean Rousseau
}

Il est des sciences, comme l'axiomatique ou la logique, qui n'ont nul besoin de pratiquer la comparaison. À l'inverse, parmi toutes celles, très nombreuses, qui y recourent, il en est pour qui l'exercice de la comparaison est au principe même de leur constitution, telle l'ethnologie quand elle apparaît dans la pensée des Lumières ou la philosophie de l'histoire avec Ibn Khaldûn. D'autres disciplines, une fois constituées, lui ménagent, tardivement dans l'histoire de leur développement, une place limitée, c'est le cas du droit, de l'histoire des religions, de l'anthropologie. Enfin, certaines sciences peuvent aussi bien pratiquer la comparaison dans le processus de constitution de leur objet comme science, parfois même sans en avoir pleine conscience réflexive, qu'en faire l'objet exclusif de leur investigation. C'est le cas de la linguistique. Celle-ci, quand elle analyse une langue pour en produire la description, d'ordre phonétique, morphologique, syntaxique, ou sémantique, use déjà constamment de procédures de type comparatif. Elle a en permanence à trancher du même et de l'autre, à asserter, quelle que soit la nature des unités considérées, sous des formes : $\mathrm{A}$ est différent de $\mathrm{B} ; \mathrm{A}$ est à $\mathrm{B}$ comme $\mathrm{C}$ est à $\mathrm{D}$ etc. À l'évidence, pratiquer commutations, permutations, substitutions, transformations, c'est toujours comparer. Quand on identifie des unités et les rapports les unissant, l'articulation de divers niveaux de fonctionnement, dès qu'on définit des catégories, regroupe des phénomènes en classes, on sollicite nécessairement l'aptitude cognitive à comparer des objets.

\section{La comparatistique}

2 Si l'on entreprenait, en conséquence, de recenser les méthodes utilisées pour l'investigation comparative dans chacun des champs disciplinaires qui font appel à elle, et qu'on les soumette elles-mêmes à la comparaison, on saisirait sans doute, dans toutes les stratégies de recherche parallèlement et indépendamment menées, des homologies 
fondamentales de cet ordre qui constitueraient une sorte d'essence de la comparaison et formeraient les premiers linéaments d'une sorte de Comparatistique générale. Mais, outre le fait qu'un tel relevé n'a jamais été vraiment tenté, il ne révélerait probablement, comme fonds commun de pratiques et de principes méthodologiques, que des évidences, telles que la quête de structures, d'invariants, de dispositifs articulés en systèmes, finalement peu éclairantes, de par le niveau d'abstraction auquel elles se situeraient.

3 Une autre direction de travail, intéressant la prise de conscience critique et épistémologique, consisterait à suivre transversalement les avatars de concepts tels que ceux de marque, de trait, de fonction, de structure, de système, différemment utilisés selon les objets disciplinaires pour lesquels on les invoque aux fins comparatives, puis, en complément, selon une perspective longitudinale, cette fois, à décrire leur développement dans diverses sciences, par échanges, transferts, extensions, réductions et détournements. On repérerait, chemin faisant, les grandes figures qui, unifiant ces concepts à contenu labile, ont successivement aimanté les recherches de type comparatiste. Celles-ci n'ont cessé d'être parcourues, et surdéterminées dans leurs applications, par des métaphores comme celle de l'organisme biologique ou les modèles fournis par la thermodynamique, la mécanique, s'inspirant de thématiques dictées par les sciences phares, aujourd'hui la théorie de l'information, la génétique etc., ou bien encore, reprenant des hypothèses explicatives empruntées aux théories à vocation holistique, l'évolutionnisme ou le matérialisme historique.

4 À toute période du développement des diverses sciences participant de l'activité comparative, l'une d'entre elles, forte de ses résultats, tend ainsi à devenir, avec ses acquis reconnus, la référence méthodologique principale et, dans des proportions variables, se met à infléchir la visée des autres disciplines qui se tournent vers elle en quête de scientificité. Sans conteste, la linguistique a joué, un temps, vers le milieu de notre siècle, ce rôle avec plus ou moins de bonheur selon les domaines d'application. On sait bien, par exemple, l'importance qu'a revêtue pour Claude Lévi-Strauss sa participation au séminaire de Roman Jakobson à New York, durant la dernière guerre, et son utilisation de la phonologie pour formaliser les structures de parenté. La linguistique tirait évidemment parti de son articulation en deux niveaux bien distincts d'unités de nature différente. La possibilité d'une segmentation, quasi spontanée pour le mot ou l'énoncé minimum, plus conceptuelle pour le phonème, apparaissait comme exceptionnelle, au regard d'autres champs d'observation, dépourvus, eux, de telle unités minimum à comparer.

5 Mais les temps sont révolus où la linguistique, avec une identité forte et des méthodes éprouvées, pouvait dicter ses lois au reste des sciences humaines, imposant la notion de structure comme norme de scientificité à rejoindre impérativement. Privée d'une doctrine de référence incontestée, elle ne se pose plus en donneuse de leçons de théorie. Son champ est désormais trop éclaté, ses doutes trop voyants, pour que les autres sciences du social en attendent encore leur paradigme, y cherchent des modèles immédiatement opératoires. Dès lors mon propos sera empreint d'une modestie, certes rituelle dans le contexte, mais particulièrement appropriée : parcourir quelques étapes du développement de la linguistique afin de retracer son expérience en matière de comparaison entendue au sens étroit. La linguistique n'a cessé en effet d'être travaillée par le projet de comparer les langues entre elles, empruntant des voies très diversifiées, et souvent parallèles, pour l'accomplir. Dégager quelques-unes de ses pratiques et de ses 
méthodes, peut donc apporter un éclairage indirect à des sciences connexes, plus novices en la matière, mais animées aujourd'hui de préoccupations analogues.

On tentera ainsi, dans les pages qui suivent, de présenter une typologie des formes que revêt la comparaison quand elle s'exerce sur des langues différentes. Si l'on prend les sciences du langage, au cours d'une histoire qui commence avec les analyses grammaticales à usage des écoliers sumériens, l'âge de la comparaison est relativement récent, deux siècles environ. Mais si, depuis ce tournant qui a inauguré un traitement proprement scientifique des langues, le comparatisme au sens classique n'a cessé de voir diminuer son importance au profit d'autres programmes de recherche plus vivaces, ces derniers n'en recourent pas moins, depuis lors, fût-ce dans l'implicite, à d'autres approches pour comparer les langues qui seront également esquissées.

\section{La comparaison génétique}

7 Remarquons tout d'abord que l'approche comparatiste n'a jamais été une condition pour que se constituent les sciences du langage, qu'elle n'est pas liée à la qualité du questionnement dont les langues ont été l'objet. Pendant des siècles, on a pu faire œuvre de linguiste sans se soucier de confronter des langues entre elles. L'ampleur de la réflexion philosophique des Grecs sur leur propre idiome qui se traduit par l'analyse aristotélicienne de la prédication, l'élaboration des catégories ou la définition des parties du discours par les Stoïciens, s'est fort bien déployée en l'absence de tout intérêt pour la langue de l'autre, du non-grec, du Barbare, même le plus proche. Des siècles d'affrontement avec les Perses n'ont pas amené les Grecs à écrire une seule ligne sur les analogies, évidentes pour nous à la simple audition, qui unissent ces deux langues parentes. La même observation vaut pour la riche tradition linguistique des Indiens ou des Arabes.

8 Pour que la conversion du regard soit possible, que la langue des confins ou des lointains devienne, avec la sienne propre, l'objet de cette «double attention» par laquelle Condillac définissait la comparaison, il faut qu'en naisse le besoin impérieux. Celui-ci, à l'évidence, n'a été vraiment éprouvé que par l'Occident chrétien. Dès que se manifeste sa dynamique d'expansion, lorsqu'il prend pied chez les autres peuples, après la maitrise de l'espace et l'établissement de l'échange inégal, le moment où il devient nécessaire d'asseoir sa domination sur ceux qu'on a déjà soumis économiquement n'est jamais très éloignée. Plus sûrement que par la simple animalisation, il importe de gagner les esprits. Pour le missionnaire qui voyage dans les fourgons du conquérant ou du marchand, il faut très vite baptiser, confesser, prêcher. En règle générale, après leur arrivée, en moins d'une génération, les plus brillants, les Jésuites, se dotent des premiers outils indispensables, vocabulaires et grammaires. De même, ce sont les grandes puissances impériales naissantes, la Russie de Pierre le Grand ou les États-Unis de Jefferson qui, un peu plus tard, lanceront de grands programmes, fort coûteux en hommes et en moyens, afin de dresser la carte des immensités à conquérir, connaître les usages des peuples à réduire, mais surtout leurs langues, et plus précisément leurs rapports mutuels, permettant une éventuelle union contre l'envahisseur blanc.

9 Longtemps, ces matériaux en provenance du monde entier s'entassent sur le bureau des savants professeurs sans effet notable. Le spectacle de l'infinie diversité des langues demeurait incompréhensible: les échantillons linguistiques sont collectionnés comme autant d'anomalies; plus proches des monstres des cabinets de sciences naturelles que 
des herbiers des botanistes, ils prennent place dans une sorte de Wunderkammer des productions intellectuelles de l'humanité. Une telle diversité est en effet, à la lettre, impensable dans l'horizon mental de la pensée classique qui est condamnée à vouloir la résorber en fonction d'un seul principe explicatif, en l'espèce inopérant, celui d'une unité originelle, sous l'égide de l'hébreu, que dicte la Genèse.

Condition nécessaire, l'accumulation primitive des documents ne suffit donc pas à imposer leur confrontation. Être acculé à la comparaison présuppose bien davantage : des questions à poser et l'exigence de réponses. En l'occurrence, l'interrogation pressante qui a précipité le déclenchement de la fièvre comparante est venue des sciences de l'histoire. Il est devenu - en gros avec Leibniz - évident que le seul moyen de résoudre des questions tournant autour de l'origine des peuples, de leurs migrations, de leurs liens, était, lorsque tout autre document faisait défaut, de recourir à l'examen des témoignages linguistiques et d'essayer les rapprochements qu'il suggérait à l'observateur. La comparaison linguistique n'a donc été initialement pratiquée selon une visée historique et génétique que dans la mesure où les langues ont été, comme en désespoir de cause, sommées de résoudre des problèmes de nature ethno-historique.

Encore fallait-il un cadre mental autorisant l'hypothèse d'une évolution des formes. En lieu et place d'une datation du Déluge très récente (- $4004 \mathrm{av.} \mathrm{J.-C.)} \mathrm{qui} \mathrm{impliquait} \mathrm{un} \mathrm{quasi}$ fixisme, la sécularisation de la vision du monde des Lumières laisse désormais penser une profondeur temporelle suffisante pour permettre une altération des langues justifiant les écarts constatés. Cette dilatation de la perspective, quoique tardive, ouvre aussitôt un espace de variation salutaire. Les langues romanes, malgré leur proximité, n'ont ainsi pu être posées en toute clarté comme autant de descendants du latin que dans les premières années du dix-neuvième siècle.

Mais c'est surtout la prégnance des acquis théoriques des sciences naturelles qui fut déterminante, avec Carl Linné qui produisit une classification exhaustive des plantes et des animaux selon une procédure rationnelle recourant à des critères simples. Les comparatistes, même dans la perspective historique, y ont vu d'emblée un modèle pour leur propre horizon de recherche; et leurs conclusions quant aux rapports entretenus par les langues du point de vue de leur origine débouchèrent très vite sur une taxinomie des langues.

Dernier point enfin, il fallait, pour lancer l'entreprise, que le modèle d'une langue-mère originelle à qui imputer, par diverses transformations, une descendance sous forme de langues différentes - rôle longtemps joué, quoique de plus en plus difficilement, par l'hébreu, puis dénié à cette langue - soit conservé, tout en étant transféré à une autre, tout aussi réputée pour son ancienneté, mais plus féconde dans son application au domaine indo-européen, à savoir le sanscrit, avant que d'être subverti au profit d'une entité imaginaire, produite par une construction théorique.

En outre, la mobilisation du corps social tout entier autour du projet comparatif, qui se traduit par des crédits, la création de chaires universitaires, serait inexplicable sans le besoin de trouver des réponses à une quête angoissée de l'origine. L'Allemagne préromantique, en particulier, en des temps où la Nation était encore purement idéale, a pu trouver là une première fondation, du point de vue de sa langue, et un enracinement inespéré dans un passé mythique, dans la figure glorieuse d'un mythe d'origine qui anticipait une unité politique rêvée. 
15 La première comparaison, dans la seconde moitié du dix-huitième siècle, s'est ainsi effectuée entre deux ou plusieurs langues par un travail sur des paires d'items simplement tirés du lexique, items qui sont ensuite déclarés ressemblants ou différents, pour leur forme et leur sens. En effet, si l'on postule les données lexicales dépourvues de motivation, puisqu'elles reposent sur des conventions sociales non explicitées, et si l'hypothèse de l'emprunt est exclue, une ressemblance à la fois sémantique et phonétique n'a, quand les exemples sont si nombreux qu'on ne puisse invoquer le hasard, d'autre explication, pour des langues géographiquement éloignées, que celle d'une identité d'origine. D'une forme unique, et par une transformation lente, sont sorties les formes aujourd'hui plus ou moins distantes que l'on observe dans deux langues posées en parallèle - qu'on pense au reflet d'un mot latin dans les langues romanes. Mais à la limite, disposer de la forme originelle est non pertinent, car l'hypothèse est assez forte pour valider des rapprochements entre langues dont la langue-source a disparu et qui, sur la seule base de paires systématiques, seront déclarées parentes, même en l'absence de tout ancêtre encore disponible.

Dans la même visée d'une comparaison des langues fondée sur la perspective historique, une nouvelle étape fut rapidement franchie. Dans les premières années du dix-neuvième siècle, avec Franz Bopp, les frères Grimm, la méthode s'émancipe sur différents points. D'abord, elle s'affranchit progressivement de l'exigence d'une ressemblance entre items. Ce n'est plus l'identité qui est le meilleur garant d'une relation probante : entre deux éléments pris dans des langues différentes la distance formelle peut être considérable (malgré les apparences, un $d w$ - du grec correspond à erk - en arménien), pourvu que l'on dispose d'autres exemples, même en très petit nombre (trois dans les derniers cas), qui manifestent exactement la même correspondance de langue à langue, car c'est la cohérence, la systématicité des rapprochements qui, bien plus que leur évidence, vaut désormais comme preuve d'une origine commune.

Ensuite, la comparaison ne s'exerce plus prioritairement sur des unités lexicales élémentaires, elle prend comme objet les faits morphologiques, en s'appuyant sur des éléments de statut inférieur au mot, exprimant des catégories grammaticales, les morphèmes, qui viennent plus ou moins dans cette période d'être reconnus. Une langue apparait désormais comme une combinatoire d'unités minimales porteuses de signification, identifiables par diverses procédures qui permettent de segmenter des unités plus complexes. En conséquence, ce sont les structures qui deviennent la matière première de la comparaison : des rapports, des proportions qui se retrouvent de langue à langue. Il suffira de repérer, en une langue nouvelle, l'alternance entre une forme pleine ${ }^{*} e s$ - et une forme réduite ${ }^{*}$ s- pour exprimer le présent singulier et pluriel (du type es- $t$ / $s$ unt, en latin, is-t / s-ind en allemand, asti / santi en sanscrit) pour déclarer, dès la découverte en hittite de eszi / asanzi, que cette langue est indoeuropéenne. Enfin, l'objectif majeur devient sur la base de telles correspondances systématiques, la reconstruction du prototype commun, aujourd'hui disparu, qui est à postuler nécessairement pour les expliquer.

18 Une discipline puissante s'est donc construite, à quoi, pour un siècle environ, s'est trouvé résumé tout l'effort scientifique de la linguistique, mobilisant équipes de recherche, chaires et étudiants, produisant manuels et grammaires comparées. Ses résultats ont été considérables puisqu'elle a pu à la fois reconstituer un passé autrement inaccessible, et surtout produire, en diversifiant ses domaines d'application jusqu'à couvrir la majeure partie du globe, une classification génétique des langues, réparties en familles 
hiérarchisées dont les contours sont aujourd'hui universellement acceptés, même si le degré d'unification atteignable et l'ampleur des regroupements possibles font toujours l'objet d'âpres litiges.

\section{La comparaison typologique}

19 Pourtant, parallèlement à cet essor remarquable d'une comparaison à visée historique, s'est aussi affirmée, d'abord discrètement, sous la plume d'auteurs comme Wilhelm von Humboldt, plus soucieux de généraliser que de traiter de faits particuliers émiettés à l'infini, un autre principe comparatif. Son projet est plus proche des objectifs comparatifs des sciences contemporaines, en ce qu'il est cette fois d'essence synchronique. Il ne s'agit plus de comparer les langues pour retracer leur filiation historique par dispersion dialectale à partir d'une origine commune reconstruite, mais de découvrir, pour chaque langue, le principe d'organisation auquel on puisse, sans la trahir, ramener l'essentiel de son fonctionnement grammatical. La difficulté d'une telle investigation a été assurément sous-estimée, et la pratique s'en est, à l'usage, avérée réductrice, puisque, le plus souvent, c'est un trait structural hégémonique que l'on se contente d'exhiber. Mais l'effort, qui s'appuyait sur la conviction de l'existence d'une forme interne individuelle structurant en chaque langue tous les phénomènes de surface, a débouché sur une classification des langues autour de quelques types selon le mode de fonctionnement des unités morphologiques par rapport au mot (langues isolantes, agglutinantes, flexionnelles, à incorporation).

20 À l'aide d'un petit nombre de traits, il devient alors théoriquement possible de définir le caractère d'une langue dans une visée qui n'a plus rien d'historique et de produire une classification strictement structurale des langues, qu'on répartit en quelques types fondamentaux. Pourtant, cette approche, plus tard étiquetée comme comparaison typologique, ne révéla pas immédiatement sa fécondité et son intérêt. En effet, elle souffrit durablement d'avoir pris naissance au sein de préoccupations principalement axées sur l'histoire. Du coup, elle en demeura tributaire et dut surtout contribuer à les justifier. Dès son établissement, cet éventail des types fut aussitôt traité de manière axiologique et posé comme ayant valeur de hiérarchie. On voulut voir dans la répartition obtenue comme une succession à validité historique : les types caractériseraient autant de stades de développement en fonction d'une évolution linéaire, qu'ils permettraient de retracer, s'avançant plus ou moins loin et plus ou moins vite le long d'un parcours linéaire obligé qui est en définitive celui du progrès global du langage humain. Ce faisant, la comparaison typologique eut le malheur d'épouser le modèle transformiste de l'évolution des espèces, certains linguistes, dans la seconde moitié du dix-neuvième siècle s'affichant sans réserve comme darwinistes.

21 Il fallut attendre les années vingt de notre siècle pour que la typologie s'affranchisse enfin de cette tutelle encombrante, retrouve ses droits et soit de nouveau abordée dans une rigoureuse synchronie. Plusieurs acquis scandent cette émancipation lancée par le grand spécialiste des langues amérindiennes que fut Edward Sapir ; ils nous mèneront jusqu'aux travaux contemporains.

22 En premier lieu, l'application de critères relativement simples n'autorisant qu'un petit nombre de cases où ranger les langues fut relayée par l'élaboration d'une multiplicité de traits de structure, chaque langue devient le produit d'une combinaison originale de plusieurs d'entre eux. À une répartition simplificatrice des langues sous quelques 
rubriques succède une caractérisation par un faisceau de critères infiniment plus propre à en dégager la complexité. Corrélativement à cet effacement des distinctions tranchées au moyen de catégorisations globales s'installe une conception qui, pour chaque critère retenu comme pertinent pour définir un aspect du fonctionnement linguistique, s'efforcera de poser une échelle définissant l'intensité de sa réalisation selon les langues qui le manifestent. On ne se contentera plus de poser l'absence ou la présence de tel ou tel trait, on définira des procédures quantitatives permettant, en fonction d'indices, de mesurer pour chaque langue ce degré variable d'incarnation.

\section{Universaux de langue et grammaire universelle}

Ce repérage de traits structuraux a conduit, depuis les années cinquante, sous l'impulsion d'un autre américain, Joseph Greenberg, à une enquête comparative d'une ampleur exceptionnelle. Idéalement exhaustive, elle travaille en pratique seulement sur des échantillons qu'on espère représentatifs des quelque 5000 langues du globe. Mais surtout son objectif s'est élargi, car l'identification transversale des traits et de leur réalisation variable a débouché sur un projet dont l'inspiration classificatrice est reléguée au second plan. Il ne s'agit plus au premier chef de trouver en quoi une langue donnée est comme certaines autres, ainsi que se le proposait la typologie morphologique, mais de découvrir dans quelle mesure une langue est comme toutes les autres, d'établir des universaux de langue. Décisive apparaît maintenant la volonté de traiter les caractéristiques reconnues - qu'elles touchent à l'inventaire phonologique, à la structure morphologique, aux procédés syntaxiques ou à l'ordre des éléments - d'après leurs combinaisons possibles et de dégager celles qui se retrouvent en toute langue. Tout l'effort consiste désormais, sur la base d'un si grand nombre d'exemples empruntés aux langues les plus diverses qu'il puisse caractériser le langage humain tout entier, à poser d'abord des assertions comme "toutes les langues ont la propriété X ", puis à mettre en évidence des implications du type : «Pour toute langue, si une langue possède la propriété $\mathrm{X}$, alors elle possèdera aussi la propriété Y ». Ainsi : «Si une langue a comme ordre syntaxique de base Verbe-SujetObjet, alors elle a des prépositions ». Ou encore : «Si une langue a le phonème $/ \mathrm{m} /$, elle a aussi /n/ »; et, en conséquence, on inférera, si l'universel est valide, la présence de /n/ de la présence de $/ \mathrm{m} /$, puisque $/ \mathrm{n} /$ peut apparaître sans $/ \mathrm{m} /$, mais non $/ \mathrm{m} /$ sans $/ \mathrm{n}$ / (dans ce cas le phonème de base, le plus fondamental, le plus marqué, est $/ \mathrm{n} /$ ).

24 Allant plus loin, on posera, dans des cas privilégiés, des chaînes d'implications, ordonnées en séquence, et, dès lors, il devient possible de montrer que des traits de structure ainsi reliés se retrouvent hiérarchisés de manière identique dans toutes les langues. Dans $P>Q>R$, on a une suite de propriétés telle qu'une propriété précédente peut apparaître sans les propriétés suivantes, alors que l'inverse n'est pas vrai ; la dernière, $R$, étant la plus marquée. En toute langue connue, ou en tout cas pour un nombre très significatif d'entre elles, la construction des relatives offre ainsi une série d'implications qui prouvent une hiérarchie dans les possibilités de former une relative à partir des syntagmes nominaux de la phrase principale, selon leur fonction : sujet > objet direct > objet indirect $>$ objet d'une préposition $>$ génitif $>$ objet de comparaison. Certaines langues, comme le malgache, limitent l'emploi des relatives à la première position, d'autres, comme l'anglais, vont jusqu'au bout (the boy who Fred is taller than), tandis que le français s'arrête à la pénultième. Mais l'important est le caractère obligé de la séquence 
ainsi posée : une langue capable de relativiser un possesseur ne saurait manquer de relativiser un objet, alors que l'inverse est possible.

Or, le domaine où de telles implications se révèlent n'est pas borné strictement à la linguistique. L'étude du traitement des couleurs par les différentes cultures du monde a longtemps piétiné, butant sur le fait que les langues ont des nombres différents de termes désignant les couleurs et que les limites sont variables entre termes désignant des couleurs adjacentes sur le spectre. En revanche, si on interroge sur le foyer d'un terme de couleur, c'est-à-dire la couleur que les locuteurs considèrent comme la plus typique, pour un terme donné, il apparaît que si les limites diffèrent selon les langues, il y accord sur le foyer. De plus, si l'on observe ces foyers du point de vue de leur nombre et de leur place dans un très grand nombre de langues, une série d'universaux implicationnels émerge nettement :

blanc $>$ rouge $>$ jaune $>$ bleu $>$ brun

Toutes les langues ont des foyers pour «blanc» et «noir"; si une langue a seulement trois termes de couleur, le troisième aura comme foyer "rouge», et si elle en a un cinquième, ce sera "jaune » et " vert », mais si elle n'en a que quatre, ce sera l'un de ces deux derniers sans préférence hiérarchique, etc. Plus abstraitement, si une langue a un terme de couleur avec le foyer $\mathrm{x}$, alors elle a aussi un terme de couleur pour chaque foyer situé à la gauche de $\mathrm{x}$ dans le diagramme.

Un tel effort pour découvrir des modes d'ordination de traits universellement valables est sans doute d'un intérêt empirique incontestable, puisque, comme dans le cas des relatives, on peut en tirer des prédictions. Et, peut-être les autres disciplines œuvrant à la comparaison y verraient-elles un exemple à suivre pour conférer une cohérence à l'organisation de leurs données. Mais il serait prématuré de poser là un modèle de la méthode comparative, tant que l'explication de telles structurations n'est pas claire. Or celle que fournissent ceux qui les proposent n'emporte pas vraiment la conviction. Elle est en effet cherchée du côté des contraintes de la cognition et de la communication : les universaux linguistiques accroîtraient la facilité avec laquelle le contenu sémantique d'un énoncé pourrait être retrouvé à partir de la structure fonctionnelle. Mais comme, par bien des aspects, la langue est traversée de dysfonctionnements, on peut douter d'une telle justification.

Pour Noam Chomsky, le but de la linguistique n'est pas de caractériser le langage comme un phénomène social extérieur à l'esprit, mais de le traiter comme une connaissance interne à l'esprit humain. Les seuls universaux sont ceux que fournit la construction d'une théorie de la grammaire universelle, dont le rôle est de déterminer quels principes tirés de la description d'une langue particulière sont des universaux linguistiques. Ils sont donc tirés de la théorie et non des données comme ceux de Greenberg. Une fois repérés en une langue donnée, et si la théorie les assigne à la faculté de langage elle-même et non à l'apprentissage de cette langue particulière, ils pourront être déclarés valides sur le témoignage d'une seule langue, sans qu'il soit nécessaire de les retrouver en toute autre. La Grammaire Universelle de Chomsky ne prétend pas que toutes les langues soient identiques, les universaux peuvent parfaitement être absents des langues, le seul réquisit absolu est qu'ils n'y soient pas enfreints. Ces principes sont donc des hypothèses 
falsifiables. Dans l'approche chomskyenne de la linguistique, le déplacement s'opère des langues vers la faculté de langage. L'aptitude à acquérir toute langue possible étant génétiquement déterminée, les propriétés inhérentes à l'esprit humain sont conçues comme un ensemble de principes généraux qui s'appliquent aux grammaires de toutes les langues du monde, un petit nombre de paramètres étant laissés libres d'être, pour une langue donnée, fixés, spécifiés de telle ou telle manière, qui suffisent à rendre compte de la diversité des langues. Le but de la Grammaire Universelle est de poser les limites à l'intérieur desquelles les langues humaines peuvent varier. La théorie est fermement étayée et l'avenir est sans doute à une intégration progressive des universaux découverts empiriquement sur la base d'enquêtes typologiques systématiques, dans le cadre explicatif du paradigme chomskyen.

Le parcours qu'on vient d'esquisser peut paraître décevant à qui en attendrait des enseignements directement applicables à des comparaisons d'un autre ordre. La linguistique ne peut offrir que ce qu'elle a elle-même vécu au cours d'une histoire déjà ancienne et tumultueuse : ses hésitations, ses revirements et ses doutes. Peut-être n'estelle cependant pas la moins bien placée, de par les résultats néanmoins obtenus chemin faisant, pour lancer à l'avenir une réflexion d'ensemble sur la méthodologie de la comparaison en général.

\section{RÉSUMÉS}

La dimension comparative en linguistique, inhérente selon l'auteur au processus de constitution de cette discipline comme science, est développée depuis le XVIII ${ }^{\mathrm{e}}$ siècle. Les principales étapes de cette réflexion et la typologie des approches qui l'ont nourrie jusqu'à nos jours sont présentées ici comme l'exemple d'un parcours méthodologique.

\section{INDEX}

Mots-clés : comparaison, linguistique comparative, linguistique, analyse comparative, langues

\section{AUTEUR}

\section{JEAN ROUSSEAU}

Centre international d'études pédagogiques (CIEP) 